\title{
TERAPIAS COGNITIVO-CONDUCTUALES DE TERCERA GENERACIÓN (TTG): LA ATENCIÓN PLENA / MINDFULNESS
}

\author{
Dra. Ana Moreno Coutiño ${ }^{1}$
}

\section{RESUMEN}

La intención del presente trabajo es reseñar el tratamiento basado en la atención plena, también conocida como Mindfulness, el cual es una de las llamadas terapias cognitivoconductuales de tercera generación (TTG). Las aportaciones de las TTG se aprecian en las terapias concretas que la constituyen, las cuales tienen como principio terapéutico el abandono de la lucha contra los síntomas y en su lugar la reorientación de la vida. Recientemente las TTG han comenzado a estudiarse en las principales universidades del mundo, así como a ser utilizadas de manera satisfactoria en diversos escenarios clínicos y en diversos países occidentales. Este tipo de terapia también ha comenzado a ser evaluada en Latino América, sin embargo su introducción en el ámbito clínico y académico ha sido más lento, quizá debido a que los principios generales de la atención plena aún no han sido suficientemente difundidos. Este trabajo recapitula las bases de las TTG y expone los principales preceptos budistas en los que se encuentra fundada la atención plena, siendo su objetivo principal el describir esta aproximación terapéutica y recapitular el estado actual de las investigaciones desarrolladas hasta el momento.

Palabras clave: Atención plena, terapias cognitivo-conductuales de tercera generación, budismo, salud.

\begin{abstract}
The purpose of this paper is to review mindfulness, which is a so-called third generation cognitive behavioral therapy (TGT). Contributions of these specific therapies are appreciated in their techniques, which have as therapeutic principle abandoning the battle against the symptoms and redirecting life instead. TGT have recently begun to be studied in major universities around the world, and have been successfully used in various clinical settings, as well as in various Western countries. This kind of therapy has also been evaluated in Latin America, but its introduction in the clinical and academic fields has been slower, perhaps because the general principles of mindfulness have not yet been sufficiently widespread. This paper summarizes the basis of TGT, describes its therapeutic approach, exposes the links between the main Buddhist precepts and mindfulness, and summarizes the current status of its research in the world.
\end{abstract}

Key words: mindfulness, cognitive behavioral therapy third generation, Buddhism, health.

\footnotetext{
${ }^{1}$ Facultad de Psicología, Universidad Nacional Autónoma de México; Correo electrónico: moca_99@yahoo.com
} 


\section{TERAPIAS COGNITIVO-CONDUCTUALES DE TERCERA GENERACIÓN (TTG)}

De acuerdo con Pérez (2006), la primera generación de la terapia conductual se sitúa en la década de 1950. Por un lado, se manifestó como una rebelión frente a la psicología clínica establecida, dominada principalmente por el psicoanálisis, y por otro, supuso la instauración de una terapia psicológica sustentada en principios científicos, dados sobre todo por la psicología del aprendizaje, con dos paradigmas básicos, el condicionamiento clásico o pavloviano y el condicionamiento operante o skinneriano. Esto favoreció la evaluación científica mediante la utilización de análisis estadísticos que permitieron hacer inferencias poblacionales.

La segunda generación de la terapia de conducta se sitúa en torno a 1970, con la terapia cognitivo-conductual (TCC); en la cual la psicología cognitiva se dedicó a estudiar el guión entre el estímulo y la respuesta, relación entendida ahora a través de la metáfora del procesamiento de la información. La TCC postula que las cogniciones (creencias, pensamientos, expectativas, atribuciones, entre otras) son las causas de los problemas emocionales y conductuales y, aun más, que cada trastorno tiene su condición por no decir déficit o disfunción cognitiva específica (Hayes, 2004a; Pérez, 2006). Las TCC, mantuvieron (y aún lo hacen) las técnicas centradas en el cambio conductual por contingencias o de primer-orden (generadas por la primera ola de terapias); sin embargo, las variables de interés por excelencia fueron trasladadas a los eventos cognitivos considerándolos, ahora, como la causa directa del comportamiento y, por tanto, transformándose el pensamiento en el objetivo principal de intervención. En este sentido, la premisa básica de las TCC sostiene que si la causa de la conducta es el pensamiento, se ha de cambiar el pensamiento para cambiar la conducta. Las principales críticas a la TCC se enfocan en que éstas pueden ser limitadas debido a su propia tendencia a estandarizar los casos, y a que se derivan de una filosofía que considera que todo aquello que genere malestar o produzca dolor ha de ser rápidamente erradicado o eliminado a través de todos los medios disponibles; especialmente, enfatizando el empleo de estrategias o técnicas de control (eliminación, supresión, evitación, sustitución, etc.) (Mañas, 2007; Tai \& Turkington, 2009).

Entre las limitaciones más importantes de las terapias de segunda generación destacan los datos experimentales, los cuales indican precisamente que los intentos de control, reducción o eliminación de los eventos privados, justamente los objetivos de intervención de estas terapias, producen paradójicamente, y en muchos de los casos, efectos contrarios o de rebote. Entre estos efectos se han descrito notables incrementos tanto en la intensidad, frecuencia, así como en la duración, e incluso, en la accesibilidad a los eventos privados no deseados (Gross \& Levenson, 1993, 1997; Gutiérrez, Luciano, Rodríguez, \& Fink, 2004; Sullivan, Rouse, Bishop, \& Johnston, 1997; Wegner \& Erber, 1992).

La tercera generación de la terapia conductual se sitúa a partir de la década de 1990, aunque se daría a conocer ampliamente hasta el 2004. Hayes (2004a), considera la tercera generación de terapias de la conducta como fundamentada en una aproximación empírica y 
enfocada en los principios del aprendizaje. Considera que la tercera ola de terapias cognitivas y conductuales es particularmente sensible al contexto y a las funciones de los fenómenos psicológicos, y no sólo a la forma, enfatizando el uso de estrategias de cambio basadas en la experiencia y en el contexto además de otras más directas y didácticas. Estos tratamientos buscan la construcción de repertorios amplios, flexibles y efectivos en lugar de tender a la eliminación de los problemas claramente definidos, resaltando cuestiones que son relevantes tanto para el clínico como para la persona. Según este autor, la tercera ola reformula y sintetiza las generaciones previas de las terapia cognitivas y conductuales y las conduce hacia cuestiones, asuntos y dominios previa y principalmente dirigidos por otras tradiciones, a la espera de mejorar tanto la comprensión como los resultados.

La emergencia de esta nueva generación de terapias psicológicas se debe tanto al desarrollo del análisis de la conducta y del conductismo radical, como a las limitaciones de la terapia cognitivo-conductual, las cuales tendrían que ver con la estandarización de los tratamientos en perjuicio de aplicaciones más flexibles y ajustadas al caso. Las aportaciones de las TTG se aprecian en las terapias concretas que la constituyen, las cuales tienen como principio terapéutico el abandono de la lucha contra los síntomas y en su lugar la reorientación de la vida. Haciendo frente al ensañamiento contra los síntomas que caracterizan a la psiquiatría biológica y en buena medida también a la terapia cognitivo-conductual (Pérez, 2006). Las TTG se refieren o conectan con procesos relacionados directamente con la aceptación psicológica; los valores, tanto de la persona como los del propio terapeuta; un proceder dialéctico durante el curso de la terapia; así como cuestiones relacionadas con la espiritualidad y la transcendencia (Mañas, 2007).

De acuerdo con Hayes (2004b) las terapias de la tercera ola buscan cumplir diversos objetivos, entre ellos: abandonar el compromiso de utilizar exclusivamente cambios de primer-orden; adoptar presupuestos básicos de corte más contextualista; utilizar estrategias de cambio más experimentales en lugar de emplear exclusivamente estrategias de cambio directas o de primer-orden; así como ampliar y modificar de forma considerable el objetivo a tratar o cambiar. Hasta el momento se desconoce si la tercera ola de terapias resultará más efectiva que la anterior. La literatura sobre las TTG es aún muy joven como para poder indicar algo, pero se cuenta con importantes indicios de que marcarán un cambio positivo en las terapias del comportamiento.

En la actualidad las principales TTG son, la Terapia de Aceptación y Compromiso (Hayes, McCurry, Afari, \& Wilson, 1991; Wilson \& Luciano, 2002), la Psicoterapia Analítica Funcional (Kohlenberg \& Tsai, 1991), la Terapia de Conducta Dialéctica (Linehan, 1993), la Terapia Conductual Integrada de Pareja (Jacobson \& Christensen, 1996), la Terapia de Activación Conductual (Jacobson, Martell \& Dimidjian, 2001), la Terapia de Reducción del Estrés Basada en la Atención Plena (REBAP) (Kabat-Zinn, 1990), la Terapia Cognitiva con base en el estar atento (Mindfulness) (Segal, Williams, \& Teasdale, 2002) y la Terapia basada en el estar atento (Mindfulness) para el trastorno de ansiedad generalizada (Evans et al., 2008). Las anteriores ocupan técnicas que no se enfocan tanto en los síntomas al sujeto, sino en modificar su experiencia subjetiva y la conciencia de ella, de tal modo que los síntomas resulten excesivos u obsoletos.

Entre las terapias antes mencionadas, sobresale la terapia de REBAP, basada en la atención 
plena/Mindfulness, la cual es una técnica que se desprende de la filosofía milenaria del budismo, es sencilla, breve, fácil de aprender y aplicar, que puede utilizarse tanto en formato grupal como individual, la cual ha demostrado su eficacia en múltiples enfermedades y entornos sanitarios, como se revisará más adelante, y resulta especialmente aceptable en atención primaria (Finucane \& Mercer, 2006). Debido a esto, a continuación se describe esta técnica de manera detallada.

\section{LA ATENCIÓN PLENA/MINDFULNESS}

Mindfulness es la acepción inglesa del concepto castellano de "atención plena", "estar atento", "conciencia plena", o "presencia plena", el cual implica que el sujeto se concentre en la tarea que está realizando en ese momento, sin que la mente divague sobre el futuro o el pasado, y sin sensación de apego o rechazo, provocando en éste energía, claridad de mente y alegría (Brown \& Ryan 2003). Si se define a la mente cómo el proceso que regula el flujo de energía y de información, la técnica de mindfulness consiste en estar atento a varios aspectos de la propia mente. Es una forma concreta de atender a las experiencias del aquí y el ahora, y a la propia naturaleza de la mente desarrollando una forma especial de atención (Siegel, 2010).

Kabat-Zinn (2003) ofrece una clara definición de dicha técnica, al especificar que ésta surge al atender intencionalmente a la experiencia propia, momento a momento, aceptándola y sin juzgarla. Desarrollando así una nueva perspectiva sobre los pensamientos y los sentimientos, en la que se les reconoce como eventos mentales y no cómo aspectos del ser o reflejos exactos de la realidad.

La práctica repetida de la atención plena permite a la persona desarrollar la habilidad de, tranquilamente apartarse de los pensamientos y sentimientos durante las situaciones de estrés en lugar de engancharse en preocupaciones ansiosas u otros patrones negativos de pensamiento, que de otra manera podrían elevarse en un ciclo de estrés reactivo (Bishop, 2002). Se logra estar presentes con nuestra experiencia, en un primer momento durante las prácticas meditativas y luego de manera extendida en la vida cotidiana.

La atención plena también se ha definido como el cultivo del darse cuenta, a través de una focalización relajada, en el surgimiento de cada momento de la experiencia (Varela, Rosch, \& Thomson, 1992). De acuerdo con estos autores, la presencia plena también significa que la mente está presente en la experiencia corpórea cotidiana. Las técnicas están diseñadas para sacar a la mente de sus teorías y preocupaciones, desde la actitud abstracta, hacia la situación concreta del "ahora y aquî" de su propia experiencia. Varela et al., (1992) además señalan que meditar y la práctica de mindfulness son en cierto sentido términos intercambiables, siendo la meditación vipassana la que mejor encaja con los modelos médicos occidentales y sobre la que existe gran evidencia en cuanto a sus efectos benéficos en múltiples trastornos. La meditación vipassana se centra en la respiración natural, observando la naturaleza cambiante de cuerpo y la mente, y así experimentar la verdades universales de la impermanencia, el sufrimiento y la ausencia del yo (Nayaka-Thera, 2003).

A través de la práctica de la atención plena, tanto en instancias formales como en la vida cotidiana, el practicante se vuelve progresivamente capaz de reconocer y observar tal y 
como son sus pensamientos, en vez de tomarlos como si fuesen hechos indiscutibles, dejando de identificarse gradualmente con sus contenidos mentales y de las emociones conflictivas asociadas, evaluando de esta manera y con mayor holgura cada circunstancia vivida, pudiendo así responder creativa y efectivamente, en lugar de reaccionar automáticamente desde sus patrones habituales (Segal et al., 2002). A los practicantes se les enseña a focalizar su atención en algo específico, como la respiración, observando con ecuanimidad el surgimiento esperable de pensamientos, emociones y/o sensaciones diversas. Cuando la persona se da cuenta de que se ha distraído, y de que su mente ha divagado, la práctica consiste en reconocer en ese momento el contenido de ese pensamiento y retornar al foco de atención. Se da un proceso circular que consiste en prestar atención plena, distraerse, hacerse consciente de la distracción, y amablemente volver a prestar atención plena. La tendencia a la distracción disminuye gradualmente a medida que aumenta la familiaridad con la práctica (Kabat-Zinn, 2003).

El cultivo de la conciencia de la mente y del cuerpo para vivir aquí y ahora es una práctica históricamente arraigada en antiguas disciplinas meditativas budistas (Stahl \& Goldstein, 2010), por lo que, de acuerdo con algunos autores, la práctica meditativa budista, en el mundo occidental, ha sido considerada más como una técnica cercana a una psicoterapia que a una religión. El budismo es una filosofía que surge alrededor del siglo $\mathrm{V}$ a. C. en el noreste de la India (Watts, 1992). A continuación se describen los principales aspectos del budismo que son la base de la atención plena.

\section{ATENCIÓN PLENA Y BUDISMO}

Las enseñanzas del budismo se centran en la mente, bajo el precepto de que una mente bien formada está tranquila y no se deja llevar por los deseos, preocupaciones o engaños. La atención plena enriquece las experiencias y hace que éstas sean satisfactorias. Una fantasía muy habitual de la mente es la de creer que todas las cosas y seres tienen una especie de existencia independiente y eterna; sin embargo, la conciencia plena expone la transitoriedad e interdependencia de los fenómenos; así mismo señala que la meditación es el método más poderoso para formar la mente. Una mente relajada y centrada permite la práctica de Vipassana o meditación concentrada. La meditación forma la mente para calmarse y observar. Al comprender que los estados de ánimo, las sensaciones y los pensamientos no duran eternamente, nuestras reacciones se calman. Además, se verá con claridad las respuestas habituales a las situaciones, lo que brinda la oportunidad de cambiar las actitudes o el comportamiento si éstos no son correctos (Gyatso, 1998). La atención plena es la más antigua de las prácticas budistas de meditación. El método viene directamente del Satipatthana Sutta, un discurso que se atribuye al mismo Buda Sakyamuni. Es un sistema antiguo y codificado de entrenamiento en sensibilidad (i. e., escuchar con atención, ver completamente y saborear con cuidado) (Vipassana Fellowship, 2012).

Los cuatro fundamentos de la atención plena, explicados en el Satipatthana Sutta (Nikaya, 2500 A. C.), son las enseñanzas originales que Buda impartió para la práctica de introspección Vipassana, y corresponden a: 1) Observar el cuerpo, lo cual significa ver aquello que hace que el cuerpo se mueva, captar la intención que impulsa a la mente a actuar; 2) Observar las sensaciones, lo cual implica prestar atención al apego a las sensaciones (i. e., agradables, desagradables o neutras); 3) Observar la mente, es decir, estar 
atento de la atracción, la repulsión y la indiferencia que aparece en el pensamiento; 4) Observar los objetos mentales como la alegría, apatía, preocupación, calma, duda, inquietud, y cualquier otra actitud mental.

La primera práctica en el camino budista de la meditación se llama "shamata" - en tibetano "shiné"-, morar en calma o "meditación de la tranquilidad." Se trata de una práctica de atención, la cual se puede realizar con un objeto (soporte) o sin él. Se basa en observar la respiración levemente y con atención. La disciplina de la práctica de shamata es hacer que la mente vuelva una y otra vez a la respiración. Según se va perfeccionando esta práctica la persona logra unificarse con la respiración, y al cabo de un tiempo, incluso la respiración, como objeto de atención de la práctica, se disuelve y se logra reposar en el momento presente. El estado de estar centrado en un único punto constituye el fruto y la finalidad de shamata (Rimpoché, 2012). Este conjunto de actividades mentales están específicamente dirigidas a experimentar un estado de conciencia (Sati) ininterrumpido. Sati es el instante de conciencia pura antes de la conceptualización, el momento de leve y desenfocada conciencia, es observación sin juicio, es la habilidad de la mente para observar sin crítica, para ver las cosas sin condenas ni calificativos. El objetivo de la meditación Vipassana es aprender a prolongar ese momento original. Al mismo tiempo, Sati hace posible el crecimiento de la sabiduría y la compasión, sin ella no sería posible desarrollar estas cualidades hasta su madurez, ya que el estado completamente desarrollado de conciencia pura es un estado de no apego y ausencia total de adicción a cualquier cosa en el mundo (Nayaka-Thera, 2003).

Para que la atención plena, Sati, pueda alcanzar su máximo desarrollo, ésta debe basarse en los principales preceptos budistas. Las cuatro nobles Verdades son los fundamentos de las enseñanzas budistas sobre los que se asientan sus distintas prácticas. La primera Verdad establece que la vida es sufrimiento. En la vida hay dolor, enfermedades y al final la muerte. También hay sufrimiento mental como el miedo, la ira, la frustración, la envidia, la decepción, etc. La segunda Verdad dice que el sufrimiento es resultado de los deseos y de la ignorancia. Llegar a Nirvana es llegar a un estado sin deseos, librarse de ese sufrimiento. La condición humana nos demuestra que un deseo cumplido puede resultar en el surgimiento de un nuevo deseo. La avaricia y el egoísmo pueden hacer que un deseo cumplido resulte en la formación de una nueva atadura, y por otro lado, un deseo no cumplido puede resultar en la ira y frustración. La tercera noble Verdad dice que se puede superar el sufrimiento y que es posible lograr la verdadera felicidad. Tenemos que concentrarnos en conocer las causas de nuestro sufrimiento, neutralizar esa ignorancia y orientar nuestra vida a superarlo. La cuarta noble Verdad establece que se puede superar el sufrimiento si uno sigue el Noble Camino Óctuple y su representación es la rueda del dharma, símbolo más universal del budismo.

De forma resumida, el Noble Camino Óctuple consiste en ser íntegro en todos los aspectos de la vida, concentrando la mente en ser totalmente consciente de nuestros pensamientos y actos así como desarrollar la sabiduría a través del entendimiento de las Nobles Verdades y mostrar la compasión hacia los demás. Los ocho aspectos principales del Sendero o Camino Óctuple se refieren a la sabiduría (i.e., 1. Visión o comprensión correcta, 2. Pensamiento o determinación correctos), la conducta ética (i.e., 3. Hablar correcto, 4. Actuar correcto, 5. Medio de vida correcto) y el entrenamiento de la mente o meditación (i.e., 6. Esfuerzo 
correcto, 7. Consciencia del momento correcta, 8. Concentración o meditación correcta) (Gyatso, 1997).

Por otro lado, en el budismo también se han postulado los Cuatro Pensamientos inconmensurables o sin límites, como ejes centrales que rigen su filosofía. A continuación se reseñarán dichos pensamientos y sus implicaciones: 1) Compasión o Bondad Amorosa: que todos los seres estén libres de sufrimiento y de sus causas (e. g., auto-engaños, confusiones y los pensamientos negativos). 2) Amor: que todos los seres posean la felicidad y sus causas, implicando las seis perfecciones (i. e., generosidad, paciencia, entusiasmo, ética, concentración y sabiduría). Asimismo, que posean una mente compasiva, que da lugar a la felicidad duradera, que no cambia. 3) Gozo o Alegría: una mente llena de Compasión y Amor, es una mente llena de alegría y de gozo. Descubrimos que nuestra mente natural es un estado de paz y regocijo, pero además de esto, notamos un estado de claridad, de lucidez, que es también, inseparable del gozo. En este estado entendemos que la base de la mente es esta condición, y que todos los seres poseen esta misma cualidad básica. Reconocemos que no hay bases para el apego o el odio, para el deseo y la aversión. 4) Igualdad o Ecuanimidad: que todos los seres permanezcan libres del apego y del rechazo hacia los otros. Se considera que todos los problemas vienen del apego y del rechazo, por lo que la ecuanimidad proporciona felicidad a la mente, la pacifica y la tranquiliza. Además con una mente relajada, se logra un cuerpo relajado y una respiración relajada (Gyatso, 1998).

Desde la precepción budista, cultivar la conciencia plena nos ayuda a vivir con profundidad cada momento de nuestra vida diaria. Todos tenemos la capacidad de estar presentes, sin embargo, aquellos que saben cómo practicarla, desarrollan una energía de atención plena mucho más poderosa y una mayor capacidad para sumirse tranquilamente en el presente. De esta manera se van logrando cambiar los hábitos negativos que surgen una y otra vez, siendo uno de los más relevantes el permitir que nuestra mente se proyecte constantemente hacia el futuro, sin vivir plena y felizmente en el presente (Nhat-Hanh, 2011).

La acción de la atención plena consiste en observar verdaderamente los propios pensamientos, "buenos" o "malos", contemplar la verdadera naturaleza de los pensamientos que puedan surgir, sin examinar el pasado ni invitar al futuro, sin permitir ningún aferramiento a las experiencias de gozo ni abrumarse por las situaciones tristes. Haciendo esto, se intenta alcanzar y mantener un estado de gran equilibrio, donde lo bueno y lo malo, la paz y la angustia, están desprovistos de verdadera identidad (Rimpoche, 1992).

El doctor en biología molecular, Matthieu Ricard (2005), va un paso más allá y sostiene que quien experimenta la paz interior no se siente ni destrozado por el fracaso, ni embriagado por el éxito. Sabe vivir plenamente esas experiencias en el contexto de una serenidad profunda y vasta, consciente de que ambas son efímeras y de que no tiene ningún motivo para aferrarse a ellas. No "decae" cuando las cosas toman un mal giro y debe hacer frente a la adversidad. No se hunde en la depresión, pues su felicidad reposa sobre sólidos cimientos. De igual manera, expone el término sukha, que en el budismo designa un estado de bienestar que nace de una mente sana y serena. Es una cualidad que sostiene e impregna cada experiencia. Sukha está estrechamente vinculado a la comprensión de la manera en 
que funciona nuestra mente y depende de nuestra forma de interpretar el mundo, pues si bien es difícil cambiar éste, sí es posible transformar la manera de percibirlo.

\section{LA PRÁCTICA DE LA ATENCIÓN PLENA EN LA CLÍNICA}

La práctica de esta técnica ha penetrado de manera sostenida la cultura occidental durante el curso del siglo XX, y su adaptación con fines terapéuticos ha encontrado un terreno fértil en la psicología de la salud y en la terapia cognitiva (Miró, 2006).

El psicoanálisis fue la primera escuela de psicología que mantuvo cierta relación con el budismo, pero fue Erich Fromm quien lo introdujo en Occidente con su libro "Budismo zen y psicoanálisis" (Fromm, Suzuki, \& DeMartino, 1960). Más tarde, la Asociación Americana de Psiquiatría manifestó, en 1977, su postura oficial ante la meditación, reconociendo su valor terapéutico y recomendando su investigación para conocer su eficacia clínica (American Psychiatric Association, 1977). El hito más importante para el desarrollo de este tipo de terapias fue la fundación, en 1979, del Center for Mindfulness, en la Universidad de Massachussets, por parte del Dr. Jon Kabat-Zinn, quien escribió la obra pionera sobre la meditación de reducción del estrés basada en el estar atento (REBAP) (Kabat-Zinn, 1990).

Hoy en día los diversos enfoques psicoterapéuticos basados en la atención plena forman una de las principales corrientes de la psicología contemporánea. Se ha reconocido que no hay salud sin salud mental, por lo que se ha propuesto que se debe de alertar sobre la necesidad de integrar a la salud mental en todos los aspectos de las políticas sociales y de salud, la planeación del sistema de salud, así como en la atención primaria y secundaria de la salud en general (Prince et al., 2007).

De manera resumida la práctica de la REBAP enseña a los participantes a reconocer y aceptar cada pensamiento y sentimiento, y luego soltarlos mientras regresa su atención a la respiración, esto mientras las personas se encuentran sentadas en una silla, o con las piernas cruzadas en el suelo, con la intención de poner atención en su respiración. Esta terapia se basa en el entrenamiento de habilidades y el modelo de psicoeducación. De manera habitual la REBAP se imparte en un periodo de 8 a 10 semanas en sesiones grupales (una por semana) de 90 minutos. Además se pide a los participantes que realicen ejercicios de meditación en sus casas, y se les otorgan CD para guiar las sesiones de meditación en casa (Bishop, 2002).

Los aspectos que se trabajan para lograr reducir el estrés mediante el «estar atento» son: comer atentamente, reconocer el momento presente, respiración atenta, paseo meditativo, observación corporal, meditar sentado, yoga atento acostado y de pie, indagación atenta para el estrés y la ansiedad, meditación en la bondad, aplicación de la "atención plena" a la vida cotidiana, ejercitar las ocho actitudes de la "atención plena" en la vida cotidiana, prestar atención al dolor, prestar atención a los hábitos, la bondad en la vida cotidiana y la escucha atenta (Stahl \& Goldstein, 2010).

En la actualidad, veinticinco años de investigación en atención plena, han documentado su 
eficacia. Esta terapia es efectiva para reducir el estrés y mejorar el estado de salud de pacientes con diversos trastornos médicos y psiquiátricos (Baer, 2003; Grossman, Nieman, Schmidt, \& Walach, 2004). Es importante anotar que la REBAP es no-sectarista y se puede utilizar en personas de cualquier religión o sin ninguna religión (Oman, Shapiro, Thoresen, Plate, \& Flinders, 2008).

El objetivo central de la práctica de la atención plena consiste en desarrollar una conciencia reflexiva de los rasgos usualmente implícitos de la propia vida mental. La capacidad de darse cuenta de tales rasgos permite estar mejor dispuestos a la transformación de hábitos cognitivos y emocionales, conduciendo hacia una conciencia más aguda, pero menos reactiva emocionalmente, de nuestro sentido biográfico de identidad que se proyecta hacia el pasado y hacia el futuro (Lutz, Slagter, Dunne, \& Davidson, 2008).

La profesionalización de la atención plena en la salud, y el interés que en el presente está recibiendo en la comunidad científica, se ve reflejada en el número de artículos publicados sobre este tema en revistas indexadas con sistema de revisión de pares. De acuerdo con una búsqueda realizada en la base de datos PubMed, en julio de 2012, entre los años 2002-2012 se publicaron 826 trabajos utilizando el término Mindfulness. Mediante esta revisión bibliográfica se pudo constatar el éxito de diversos tratamientos que emplean la atención plena en una amplia variedad de trastornos psicológicos tales como estrés, ansiedad, sintomatología depresiva y síndrome de estrés postraumático (Chiesa \& Serretti, 2009; Deyo, Wilson, Ong, \& Koopman, 2009; Evans et al., 2008; Flugel-Colle et al., 2010; Geary \& Rosenthal, 2011; Goldin \& Gross, 2010; Goldin, Ziv, Jazaieri, Hahn, \& Gross, 2012; Kearney, McDermott, Malte, Martinez, \& Simpson, 2012; Kimbrough, Magyari, Langenberg, Chesney, \& Berman, 2010; Klainin-Yobas, Cho, \& Creedy, 2012; Krusche, Cyhlarova, King, \& Williams, 2012; Matousek, Pruessner, \& Dobkin, 2011; Smith et al., 2008).

La efectividad de la atención plena ha sido demostrada en la mejora del estado de ánimo general, la calidad de vida, el estado de salud general, la atención, el bienestar psicológico en pacientes con cáncer y sus acompañantes, así como en diversas sintomatologías psiquiátricas (Biegel, Brown, Shapiro, \& Schubert, 2009; Birnie, Garland, \& Carlson, 2010; Campbell, Labelle, Bacon, Faris, \& Carlson, 2012; Carlson, Speca, Faris, \& Patel, 2007; Carmody, Reed, Kristeller, \& Merriam, 2008; Garland, Tamagawa, Todd, Speca, \& Carlson, 2012; Grossman et al., 2004; Hoffman et al., 2012; Jha, Krompinger, \& Baime, 2007; Ledesma \& Kumano, 2009; Ott, Norris, \& Bauer-Wu, 2006).

De igual manera se han registrado resultados exitosos en la aplicación de la atención plena en el tratamiento de diversos padecimientos físicos crónicos como: fibromialgia, colitis, hipertensión arterial, incontinencia urinaria, trastornos del sueño, sobrepeso, obesidad, dolor general, síntomas de la menopausia, dolor de espalda, tabaquismo; e incluso en la atención de los efectos secundarios al tratamiento de VIH (Baker, Costa, \& Nygaard, 2012; Brand, Holsboer-Trachsler, Naranjo, \& Schmidt, 2012; Carmody et al., 2011; Daubenmier et al., 2011; Davis, Fleming, Bonus, \& Baker, 2007; Duncan et al., 2012; Esmer, Blum, Rulf, \& Pier, 2010; Goldstein, Josephson, Xie, \& Hughes, 2012; Gross et al., 2011; Grossman, Tiefenthaler-Gilmer, Raysz, \& Kesper, 2007; Kearney, McDermott, Martínez, \& Simpson, 2011; Lush et al., 2009; Ong \& Sholtes, 2010; Sampalli, Berlasso, Fox, \& 
Petter, 2009; Sephton et al., 2007; Simpson \& Mapel, 2011; Winbush, Gross, \& Kreitzer, 2007; Wong et al., 2011; Zernicke et al., 2012).

Desde el área de las neurociencias, se sabe que en términos generales los efectos de la práctica del "estar atento" sobre el sistema nervioso se relacionan con la promoción de un estado de relajación del sistema nervioso periférico y la activación del sistema nervioso central (Creswell, Way, Eisenberger, \& Lieberman, 2007). Además, se ha reportado que el entrenamiento en atención plena mejora la conectividad cerebral (Kilpatrick et al., 2011), y provoca un incremento de la materia gris de las áreas del cerebro involucradas en los procesos de aprendizaje, memoria y regulación de las emociones (Hölzel et al., 2011).

Una posible explicación neurológica sobre los efectos de la práctica de la atención plena señala que el "estar atento" incrementa la regulación cortical pre frontal del afecto, mediante la modificación del etiquetado de los estímulos emocionalmente displacenteros, dicha modificación disminuye el afecto displacentero y mejora la salud psicológica en general de los individuos que han desarrollado el "estar atento" (Creswell, Way, Eisenberger, \& Lieberman, 2007).

Algunos estudios electroencefalográficos han revelado un incremento significativo en la actividad alfa y theta durante la meditación, lo cual es congruente con los estudios de neuroimagen que demuestran que durante la práctica de la atención plena se activan la corteza prefrontal y la corteza cingulada anterior; mientras que la práctica a largo plazo se asocia con una mejora en las áreas cerebrales relacionadas con la atención (Chiesa \& Serretti, 2010).

Por otro lado, Hölzel et al., (2010) reportaron que la reducción del estrés percibido se correlaciona con cambios estructurales en la amígdala; específicamente con decrementos en la densidad de la materia gris de la amígdala basolateral derecha. Estudios previos habían reportado que las modificaciones en la materia gris se derivan de la adquisición de: información abstracta, habilidades motoras y lenguaje. En este sentido, se demostró que los cambios neuroplásticos se asocian con mejoras en el estado de una variable psicológica; siendo prometedores dichos resultados en la eficacia de la práctica de la atención plena.

Los resultados de las investigaciones realizadas por los científicos del Laboratorio de Neurociencia Afectiva de la Universidad de Wisconsin (EE. UU.), dirigido por el Dr. Richard J. Davidson, señalan que el origen de la sensación de felicidad o de infelicidad está en la plasticidad del cerebro. Es decir, la capacidad humana de modificar físicamente el cerebro radica en los pensamientos que elegimos tener. Al igual que los músculos del cuerpo, el cerebro desarrolla y fortalece las neuronas que más utilizamos y atrofia o debilita las neuronas que menos utilizamos. Una mayor cantidad de pensamientos negativos provoca una mayor actividad en el córtex derecho del cerebro (lóbulo prefrontal derecho) lo que genera emociones no placenteras (ansiedad, depresión, envidia, sentimiento de culpa, hostilidad hacia los demás, entre otros). En otras palabras, infelicidad autogenerada. Por el contrario, a más pensamientos positivos, mayor actividad en el córtex izquierdo del cerebro (lóbulo prefrontal izquierdo) elevando las emociones placenteras y la felicidad (Davidson et 
al., 2003; Lutz, Brefczynski-Lewis, Johnstone, \& Davidson, 2008).

Los trabajos sobre la felicidad desarrollados por el equipo del Dr. Davidson, se fundamentan en tres premisas básicas, a saber: que el cerebro es un órgano en constante evolución y, por lo tanto, moldeable (plasticidad de la mente); que la relación entre el córtex cerebral izquierdo y el derecho puede ser medida y que la relación entre ambos sirve para representar el temperamento de una persona. Los estudios de resonancia magnética llevados a cabo en dicho laboratorio con monjes budistas que practican la meditación diariamente, inclu yendo al doctor en biología molecular y monje budista Matthieu Ricard, han mostrado una actividad superior inusual en el córtex izquierdo, lo cual es significativo considerando que los mayores registros de felicidad fueron detectados en dichos sujetos. Debido a lo anterior se ha sugerido que la felicidad es algo que se puede aprender, desarrollar, entrenar, mantener en forma y alcanzar definitivamente (Brefczynski-Lewis, Lutz, Schaefer, Levinson, \& Davidson, 2007).

\section{CONCLUSIÓN}

Con base en lo reseñado en este trabajo, se puede apreciar que las terapias cognitivoconductuales de tercera generación vienen a suceder a las terapias conductuales de exposición y condicionamiento operante, a las técnicas dinámicas de logro de insight, a las terapias humanistas y a las puramente cognitivo-conductuales. Dicha sucesión se da en el sentido de integrar en cierta forma a todas sus antecesoras, trabajando con un paradigma muy distinto, ya que no se trata tanto de "limpiar" de síntomas al sujeto sino de modificar su experiencia subjetiva y la conciencia que tiene de ella, de tal modo que los síntomas resulten obsoletos o irrelevantes (Hayes, 2004a).

La práctica de la atención plena permite gradualmente a las personas darse cuenta claramente de lo que está sucediendo en el momento presente, es decir observar y experimentar sin reaccionar, lo cual les permite percibir directamente la información sensorial sin analizarla, compararla o interpretarla. Esta técnica también estabiliza, sosiega y fortalece la mente y la capacita para investigar directamente la naturaleza cambiante de la vida. Las personas que logran estar plenamente atentos en el mismo instante en que se ven asaltados por el miedo, la confusión, el apego o por grandes sufrimientos, podrán valorar con detalle la situación y recuperar rápidamente el equilibrio.

La atención plena/Mindfulness utiliza técnicas desarrolladas a partir de la meditación budista, la cual a su vez parte de una filosofía milenaria que sólo recientemente se ha sometido al escrutinio científico (Kabat-Zinn, 2003). Tras los hallazgos brevemente reseñados en el presente trabajo relacionados con la eficacia clínica se puede señalar que dicha práctica meditativa es una técnica más cercana a una psicoterapia que a una mera teoría filosófica.

De acuerdo con Hayes (2004b) la evidencia recopilada hasta el momento indica que ha llegado una nueva generación de terapias cognitivas y del comportamiento, y cualquiera que sea el resultado de estos cambios, el proceso indica una maduración de las terapias del comportamiento. La cual parece que tendrá repercusiones a largo plazo muy positivas en el campo de la salud mental, adicciones, y los aspectos psicológicos y fisiológicos de las enfermedades. 
Estas técnicas han sido ampliamente estudiadas en Occidente y pueden resultar de mucho interés para los países de Latinoamérica, en los cuales la prevención primaria y la atención de segundo nivel para numerosos trastornos resultan prioritarias. Las TTG no requieren de gran infraestructura para su implementación (únicamente se requiere de un salón), por lo que no resultan costosas; además, las terapias toman sólo alrededor de ocho semanas y pueden ser impartidas por diversos profesionales del área de salud que hayan recibido la capacitación necesaria. Debido a esto, la investigación de los efectos preventivos y terapéuticos de las TTG merece seguir siendo desarrollada en poblaciones y circunstancias específicas, especialmente en Latinoamérica, ya que aunque actualmente hay numerosa evidencia sobre su capacidad de inducir cambios muy positivos en el funcionamiento del sistema nervioso y sobre su efectividad como terapia para ciertos tipos de trastornos físicos y psicológicos, aún queda mucho que investigar.

Por último, es importante mencionar que al trabajar con las TTG que se basan en la filosofía budista, la cual generalmente es muy distinta y ajena a la nuestra, se debe cuidar tener presentes los principios filosóficos que subyacen a estas terapias, ya que de lo contrario se corre el riesgo de hacer interpretaciones erróneas que ocasionarían que a la larga las TTG no dieran resultados óptimos.

\section{REFERENCIAS BIBLIOGRÁFICAS}

American Psychiatric Association. (1977). Position statement on meditation. The American Journal of Psychiatry, 134, 720-720.

Baer, R. A. (2003). Mindfulness training as a clinical intervention: A conceptual and empirical review. Clinical Psychology: Science \& Practice, 10, 125-143.

Baker, J., Costa, D., \& Nygaard, I. (2012).Mindfulness-based stress reduction for treatment of urinary urge incontinence: a pilot study. Female Pelvic Medicine \& Reconstructive Surgery, 18(1), 46-49.

Biegel, G. M., Brown, K. W., Shapiro, S. L., \& Schubert, C. M. (2009). Mindfulness-based stress reduction for the treatment of adolescent psychiatric outpatients: A randomized clinical trial. Journal of Consulting and Clinical Psychology, 77(5):855-866.

Birnie, K., Garland, S. N., \& Carlson, L. E. (2010). Psychological benefits for cancer patients and their partners participating in mindfulness-based stress reduction (MBSR). Psychooncology, 19(9), 1004-1009.

Bishop, S. R. (2002). What do we really know about mindfulness bases stress reduction? Psychosomatic Medicine, 64, 71-83.

Brand, S., Holsboer-Trachsler, E., Naranjo, J. R., \& Schmidt, S. (2012). Influence of mindfulness practice on cortisol and sleep in long-term and short-term meditators. Neuropsychobiology, 65(3), 109-118.

Brefczynski-Lewis, J.A., Lutz, A., Schaefer, H. S., Levinson, D. B., \& Davidson, R. J. (2007). Neural correlates of attentional expertise in long-term meditation practitioners. Proceedings of the National Academy of Science of the United States of America, 104(27) 11483-11488.

Brown, K., \& Ryan, R. M. (2003).The benefits of being present: mindfulness and its role in psychological well-being. Journal of Personality and Social Psychology. 84(4), 822848. 
Campbell, T. S., Labelle, L. E., Bacon, S. L., Faris, P., \& Carlson, L. E. (2012).Impact of Mindfulness-Based Stress Reduction (MBSR) on attention, rumination and resting blood pressure in women with cancer: A waitlist-controlled study. Journal of Behavior Medicine, 35(3), 262-271.

Carlson, L. E., Speca, M., Faris, P., \& Patel, K. D. (2007). One year pre-post intervention follow-up of psychological, immune, endocrine and blood pressure outcomes of mindfulness-based stress reduction (MBSR) in breast and prostate cancer outpatients. Brain, Behavior, and Immunity, 21(8), 1038-1049.

Carmody, J., Crawford, S., Salmoirago-Blotcher, E., Leung, K., Churchill, L., \& Olendzki, N. (2011). Mindfulness training for coping with hot flashes: Results of a randomized trial. Menopause, 18(6), 611-620.

Carmody, J., Reed, G., Kristeller, J., \& Merriam, P. (2008). Mindfulness, spirituality, and health-related symptoms, Journal of Psychosomatic Research, 64(4), 393-403.

Chiesa, A., \& Serretti, A. (2009). Mindfulness-based stress reduction for stress management in healthy people: a review and meta-analysis. Journal of Alternative and Complementary Medicine, 15(5), 593-600.

Chiesa, A., \& Serretti, A. (2010). A systematic review of neurobiological and clinical features of mindfulness meditations. Psychological Medicine, 40(8), 1239-1252.

Creswell, J. D., Way, B. M., Eisenberger, N. I., \& Lieberman, M. D. (2007). Neural correlates of dispositional mindfulness during affect labelling. Psychosomatic Medicine, 69(6), 560-565.

Daubenmier, J., Kristeller, J., Hecht, F. M., Maninger, N., Kuwata, M., Jhaveri, K., Lustig, R. H., Kemeny, M., Karan, L., \& Epel, E. (2011). Mindfulness intervention for stress eating to reduce cortisol and abdominal fat among overweight and obese women: An Exploratory Randomized Controlled Study. Journal of Obesity, 2011, 1-13. doi: $10.1155 / 2011 / 651936$

Davidson, R.J., Kabat-Zinn, J., Schumacher, J., Rosenkranz, M., Muller, D., Santorelli, S.F., Urbanowski, F., Harrington, A., Bonus, K., \& Sheridan, J.F. (2003). Alterations in brain and immune function produced by mindfulness meditation. Psychosomatic Medicine, 65(4), 564-570.

Davis, J. M., Fleming, M. F., Bonus, K. A., \& Baker, T. B. (2007). A pilot study on mindfulness based stress reduction for smokers. BMC Complementary and Alternative Medicine, 7(2), 1-7. doi: 10.1186/1472-6882-7-2.

Deyo, M., Wilson, K. A., Ong, J., \& Koopman, C. (2009). Mindfulness and rumination: Does mindfulness training lead to reductions in the ruminative thinking associated with depression? Explore: The Journal of Science and Healing, 5(5), 265-271.

Duncan, L. G., Moskowitz, J. T., Neilands, T. B., Dilworth, S. E., Hecht, F. M., \& Johnson, M. O. (2012). Mindfulness-based stress reduction for HIV treatment side effects: A randomized, wait-list controlled trial. Journal of Pain and Symptom Management, 43(2), 161-171.

Esmer, G., Blum, J., Rulf, J., \& Pier, J. (2010). Mindfulness-based stress reduction for failed back surgery syndrome: A randomized controlled trial. The Journal of American Osteopathic Association, 110(11), 646-652.

Evans, S., Ferrando, S., Findler, M., Stowell, C., Smart, C., \& Haglin, D. (2008). Mindfulnessbased cognitive therapy for generalized anxiety disorder. Journal of Anxiety Disorders, 22(4), 716-721. 
Finucane, A., \& Mercer, S. W. (2006). An exploratory mixed methods study of the acceptability and effectiveness of mindfulness-based cognitive therapy for patients with active depression and anxiety in primary care. BMC Psychiatry, 6(14), 1-14.

Flugel-Colle, K. F., Vincent, A., Cha, S. S., Loehrer, L. L., Bauer, B. A., \& Wahner-Roedler, D. L. (2010). Measurement of quality of life and participant experience with the mindfulness-based stress reduction program. Complementary Therapies in Clinical Practice, 16(1), 36-40.

Fromm, E., Suzuki, D. T., \& DeMartino, R. (1960). Zen Buddhism and Psychoanalysis. Nueva York: Harper \& Row.

Garland, S. N., Tamagawa, R., Todd, S. C., Speca, M., \& Carlson, L. E. (2012). Increased mindfulness is related to improved stress and mood following participation in a mindfulness-based stress reduction program in individuals with cancer. Integrative Cancer Therapies. doi: 10.1177/1534735412442370.

Geary, C., \& Rosenthal, S. L. (2011). Sustained impact of MBSR on stress, well-being, and daily spiritual experiences for 1 year in academic health care employees. Journal of Alternative and Complementary Medicine, 17(10), 939-944.

Goldin, P. R., \& Gross, J. J. (2010). Effects of mindfulness-based stress reduction (MBSR) on emotion regulation in social anxiety disorder. Emotion, 10(1), 83-91.

Goldin, P., Ziv, M., Jazaieri, H., Hahn, K., \& Gross, J. J. (2012). MBSR vs. aerobic exercise in social anxiety: fMRI of emotion regulation of negative self-beliefs. Social Cognitive and Affect Neuroscience. doi: 10.1093/scan/nss054.

Goldstein, C. M., Josephson, R., Xie, S., \& Hughes, J. W. (2012). Current perspectives on the use of meditation to reduce blood pressure. International Journal of Hypertension, 2012, 1-11.

Gross, C. R., Kreitzer, M. J., Reilly-Spong, M., Wall, M., Winbush, N. Y., Patterson, R., Mahowald, M., \& Cramer-Bornemann, M. (2011). Mindfulness-based stress reduction versus pharmacotherapy for chronic primary insomnia: A randomized controlled clinical trial. Explore: The Journal of Science and Healing, 7(2), 76-87.

Gross, J. J., \& Levenson, R. W. (1993). Emotional suppression: Physiology, self-report, and expressive behavior. Journal of Personality and Social Psychology, 64(6), 970-986.

Gross, J. J., \& Levenson, R. W. (1997). Hiding feelings: The acute effects of inhibiting negative and positive emotion. Journal of Abnormal Psychology, 106(1), 95-103.

Grossman, P., Niemann, L., Schmidt, S., \& Walach, H. (2004). Mindfulness-based stress reduction and health benefits: A meta-análisis. Journal of Psychosomatic Research, 57(1), 35-43.

Grossman, P., Tiefenthaler-Gilmer, U., Raysz, A., \& Kesper, U. (2007). Mindfulness training as an intervention for fibromyalgia: Evidence of post-intervention and 3-year follow-up benefits in well-being. Psychotherapy and Psychosomatics, 76(4), 226-233.

Gutiérrez, O., Luciano, M. C., Rodríguez, M., \& Fink, B. (2004). Comparison between an acceptance-based and a cognitive-control-based protocol for doping with pain. Behavior Therapy, 35, 767-783.

Gyatso, T. (S.S. el XIV Dalai Lama). (1997). Las cuatro nobles verdades. Los fundamentos de las enseñanzas budistas y de su correcta práctica. México: Debolsillo.

Gyatso, T. (S.S. el XIV Dalai Lama). (1998). El mundo del budismo Tibetano. Visión general de su filosofía y su práctica. Barcelona: Liberduplex.

Hayes, S. C. (2004a). Acceptance and commitment therapy and the new behavior therapies. En 
S. C. Hayes, V. M. Follette \& M. M. Linehan (Eds.), Mindfulness and acceptance: Expanding the cognitive behavioral tradition (pp. 1-29). Nueva York: The Guilford Press.

Hayes, S. C. (2004b). Acceptance and commitment therapy, relational frame theory, and the third wave of behavioral and cognitive therapies. Behavior Therapy, 35(4), 639-665.

Hayes, S. C., McCurry, S. M., Afari, N. \& Wilson, K. (1991). Acceptance and commitment therapy $(A C T)$. A therapy manual for the treatment of emotional avoidance. Reno, Nevada: Context Press.

Hoffman, C. J., Ersser, S. J., Hopkinson, J. B., Nicholls, P. G., Harrington, J. E., \& Thomas, P. W. (2012). Effectiveness of mindfulness-based stress reduction in mood, breast- and endocrine-related quality of life, and well-being in stage 0 to III breast cancer: A randomized, controlled trial. Journal of Clinical Oncology, 30(12), 1335-1342.

Hölzel, B. K., Carmody, J., Evans, K. C., Hoge, E. A., Dusek, J. A., Morgan, L., Pitman, R. K., \& Lazar, S. W. (2010). Stress reduction correlates with structural changes in the amygdala. Social Cognitive and Affective Neuroscience, 5(1), 11-17.

Hölzel, B. K., Carmody, J., Vangel, M., Congleton, C., Yerramsetti, S. M., Gard, T., \& Lazar, S. W. (2011). Mindfulness practice leads to increases in regional brain gray matter density. Psychiatry Research, 191(1), 36-43.

Jacobson, N. S., \& Christensen, A. (1996). Integrative couple therapy: Promoting acceptance and change. NuevaYork: Norton.

Jacobson, N. S., Martell, C. R., \& Dimidjian, S. (2001). Behavioral activation treatment for depression: Returning to contextual roots. Clinical Psychology: Science and Practice, 8(3), 255-270.

Jha, A. P., Krompinger, J., \& Baime, M. J. (2007). Mindfulness training modifies subsystems of attention. Cognitive, Affective and Behavior Neuroscience, 7(2), 109-119.

Kabat-Zinn, J. (1990). Full catastrophe living. Using the wisdom of your body and mind to face stress, pain, and illness. Reino Unido: Delta.

Kabat-Zinn, J. (2003). Mindfulness-based interventions in context: Past, present, and future. Clinical Psychology: Science and Practice, 10(2), 144-156.

Kearney, D. J., McDermott, K., Malte, C., Martinez, M., \& Simpson, T. L. (2012). Association of participation in a mindfulness program with measures of PTSD, depression and quality of life in a veteran sample. Journal of Clinical Psychology, 68(1), 101-116.

Kearney, D. J., McDermott, K., Martinez, M., \& Simpson, T. L. (2011).Association of participation in a mindfulness programme with bowel symptoms, gastrointestinal symptom-specific anxiety and quality of life. Alimentary Pharmacology and Therapeutics, 34(3), 363-373.

Kilpatrick, L. A., Suyenobu, B. Y., Smith, S. R., Bueller, J. A., Goodman, T., Creswell, J. D., Tillisch, K., Mayer, E. A., \& Naliboff, B. D. (2011). Impact of mindfulness-based stress reduction training on intrinsic brain connectivity. Neuroimage, 56(1), 290-298.

Kimbrough, E., Magyari, T., Langenberg, P., Chesney, M., \& Berman, B. (2010). Mindfulness intervention for child abuse survivors. Journal of Clinical Psychology, 66(1), 17-33.

Klainin-Yobas, P., Cho, M. A., \& Creedy, D. (2012). Efficacy of mindfulness-based interventions on depressive symptoms among people with mental disorders: a metaanalysis. International Journal of Nursery Studies, 49(1), 109-121.

Kohlenberg, R. J., \& Tsai, M. (1991). Functional analytic psychotherapy. Creating intense and curative therapeutic relationships. New York: Plenum Press. 
Krusche, A., Cyhlarova, E., King, S., \& Williams, J. M. G. (2012). Mindfulness online: a preliminary evaluation of the feasibility of a web-based mindfulness course and the impact on stress. BMJ Open. doi: 10.1136/bmjopen-2011-000803.

Ledesma, D., \& Kumano, H. (2009). Mindfulness-based stress reduction and cancer: A metaanalysis. Psycho-oncology, 18(6), 571-579.

Linehan, M. M. (1993). Cognitive-behavioral treatment of borderline personality disorder. New York: The Guilford Press.

Lush, E., Salmon, P., Floyd, A., Studts, J. L., Weissbecker, I., \& Sephton, S. E. (2009). Mindfulness meditation for symptom reduction in fibromyalgia: psychophysiological correlates. Journal of Clinical Psychology in Medical Settings, 16(2), 200-207.

Lutz, A., Brefczynski-Lewis, J., Johnstone, T., \& Davidson, R. J. (2008). Regulation of the neural circuitry of emotion by compassion meditation: Effects of meditative expertise. Plos One, 3(3), 1-10.

Lutz, A., Slagter, H., Dunne, J., \& Davidson, R. (2008). Attention regulation and monitoring in meditation. Trends in Cognitive Sciences, 12(4), 163-169.

Mañas, I. (2007). Nuevas terapias psicológicas: la tercera ola de terapias de conducta o terapias de tercera generación. Gaceta de Psicología, 40, 26-34.

Matousek, R. H., Pruessner, J. C., \& Dobkin, P. L. (2011). Changes in the cortisol awakening response (CAR) following participation inmindfulness-based stress reduction in women who completed treatment for breast cancer. Complementary Therapies in Clinical Practice, $17(2), 65-70$.

Miró, M. (2006). La atención plena (mindfulness) como intervención clínica para aliviar el sufrimiento y mejorar la convivencia. Revista de Psicoterapia, 17(66-67), 31-76.

Nayaka-Thera, H. G. (2003). El cultivo de la atención plena. La práctica de la meditación Vipassana. México: Editorial Pax México.

Nhat-Hanh, T. (2011). Peace is every breath: A practice for our busy lives. Reino Unido: Harper Collins Publishers.

Nikaya, M. (2500 A.C.). Satipatthana Sutta, Sutta $n^{\circ} 10$. El Sutra de los fundamentos de la atención. Recuperado de http://www.librosbudistas.com/descargas/SATI.htm

Oman, D., Shapiro, S. L., Thoresen, C. E., Plante, T. G., \& Flinders, T. (2008). Meditation lowers stress and supports forgiveness among college students: A randomized controlled trial. Journal of American Collage Health, 56(5), 569-578.

Ong, J., \& Sholtes, D. (2010). A mindfulness-based approach to the treatment of insomnia. Journal of Clinical Psychology, 66(11), 1175-1184.

Ott, M. J., Norris, R. L., \& Bauer-Wu, S. M. (2006). Mindfulness meditation for oncology patients: A discussion and critical review. Integrative Cancer Therapies, 5(2), 98-108.

Pérez, A. M. (2006). La terapia de conducta de tercera generación. Revista de psicología y psicopedagogía, 5(2), 159-172.

Prince, M., Patel, V., Saxena, S., Maj, M., Maselko, J., Phillips, M. R., Rahman, A. (2007). No health without mental health. Lancet, 370(9590), 859-877.

Ricard, M. (2005). En defensa de la felicidad. Barcelona: Urano.

Rimpoché, S. (2012). La gran paz natural. Recuperado el 10 de octubre de 2012 de http://www.budismotibetano.net/portal/index.php/tesoros-del-dharma-textos-34/enseastextos-52/273-la-gran-paz-natural-por-sogyal-rimpoch

Rimpoche, S. (1992). The Tibetan book of living and dying. San Francisco: HarperOne.

Sampalli, T., Berlasso, E., Fox, R., \& Petter, M. (2009). A controlled study of the effect of a 
mindfulness-based stress reduction technique in women with multiple chemical sensitivity, chronic fatigue syndrome, and fibromyalgia. Journal of Multidisciplinary Health, 7(2), 53-59.

Segal, Z. V., Williams, J. M. G., \& Teasdale, J. D. (2002). Mindfulness-based cognitive therapy for depression: A new approach to relapse prevention. Nueva York: Guilford Press.

Sephton, S. E., Salmon, P., Weissbecker, I., Ulmer, C., Floyd, A., Hoover, K., \& Studts, J. L. (2007). Mindfulness meditation alleviates depressive symptoms in women with fibromyalgia: results of a randomized clinical trial. Arthritis and Rheumatism, 57(1), 77-85.

Siegel, D. J. (2010). Cerebro y mindfulness. Barcelona, España: Paidós.

Simpson, J., \& Mapel, T. (2011). An investigation into the health benefits of mindfulnessbased stress reduction (MBSR) for people living with a range of chronic physical illnesses in New Zealand. The New Zealand Medical Journal, 124(1338), 68-75.

Smith, B. W., Shelley, B. M., Dalen, J., Wiggins, K., Tooley, E., \& Bernard, J. (2008). A pilot study comparing the effects of mindfulness-based and cognitive-behavioral stress reduction. Journal of Alternative and Complementary Medicine, 14(3), 251-258.

Stahl, B., \& Goldstein, E. (2010). Mindfulness para reducir el estrés. Barcelona, España: Kairós.

Sullivan, M. J. L., Rouse, D., Bishop, S., \& Johnston, S. (1997). Thought suppression, catastrophizing and pain. Cognitive Therapy and Research, 21(5), 555-568.

Tai, S., \& Turkington, D. (2009). The evolution of cognitive behavior therapy for schizophrenia: Current practice and recent developments. Schizophrenis Bulletin, 35(5), 865-873.

Varela, F. J., Rosch, E, \& Thompson, E. (1992). De cuerpo presente: las ciencias cognitivas y la experiencia humana. España: Gedisa.

Vipassana Fellowship (2012). Meditation Resourses. Recuperado el 10 de septiembre de 2012, de http://www.vipassana.com/

Watts, A. (1992). Psicoterapia del Este, Psicoterapia del Oeste. Buenos Aires: Kairos.

Wegner, D. M., \& Erber, R. (1992). The hypéraccessibility of suppressed thoughts. Journal of Personality and Social Psychology, 63(6), 903-912.

Wilson, K. G., \& Luciano, M. C. (2002). Terapia de aceptación y compromiso (ACT). Un tratamiento conductual orientado a los valores. Madrid: Pirámide.

Winbush, N. Y., Gross, C. R., \& Kreitzer, M. J. (2007).The effects of mindfulness-based stress reduction on sleep disturbance: a systematic review. Explore: The Journal of Science and Healing, 3(6), 585-591.

Wong, S. Y., Chan, F. W., Wong, R. L., Chu, M. C., Kitty-Lam, Y. Y., Mercer, S. W., \& Ma, S. H. (2011). Comparing the effectiveness of mindfulness-based stress reduction and multidisciplinary intervention programs for chronic pain: A randomized comparative trial. The Clinical Journal of Pain, 27(8), 724-734.

Zernicke, K. A., Campbell, T. S., Blustein, P. K., Fung, T. S., Johnson, J. A., Bacon, S. L., \& Carlson, L. E. (2012). Mindfulness-based stress reduction for the treatment of irritable bowel syndrome symptoms: A randomized wait-list controlled trial. International Journal of Behavioral Medicine. doi: 10.1007/s12529-012-9241-6. 
(c) (i)

Este texto está protegido por una licencia Creative Commons $\underline{4.0}$.

Usted es libre para Compartir - copiar y redistribuir el material en cualquier medio o formato- y Adaptar el documento - remezclar, transformar y crear a partir del material - para cualquier propósito, incluso comercialmente, siempre que cumpla la condición de:

Atribución: Usted debe reconocer el crédito de una obra de manera adecuada, proporcionar un enlace a la licencia, e indicar si se han realizado cambios. Puede hacerlo en cualquier forma razonable, pero no de forma tal que sugiera que tiene el apoyo del licenciante o lo recibe por el uso que hace. 